sponge bath, reading a book or mental exercise, would instantly cause a marked albumenuria. At no time during six weeks' observation could the writer detect casts, or prove that the kidneys were otherwise affected than to allow from different causes albumen to transude. A re-examination after a lapse of six months showed the same condition.

So also casts, especially the hyaline, may be present in the urine for a long time without thereby proving a serious lesion of the kidneys. It is possible that casts may, under certain conditions, form in normal kidneys.

The writer has recently had occasion to examine the urine of a gentleman who has a considerable number of casts, hyaline or slightly granular, coming only after severe exercise. The urine is normal in quantity, specific gravity, and has not contained albumen. The writer has examined the urine passed at other times of the same day on several occasions with negative results, except the presence of mucus and occasionally mucous casts. Only too frequently we are apt to alarm a patient by the detection of what may be considered a sign of imperfect action, yet not necessarily of confirmed or incurable disease, and to pronounce them afflicted with a malady whose very name is a terror.

These illustrations and remarks will suffice for the end had in view, though the same line of consideration could be continued indefinitely.

\section{THE USE OF ANTISEPTICS IN PUERPERAL CASES.}

BY MADISON REF.CE, M.D., OF ABINGDON, ILL.

[Read before the Military Tract Medical Association.]

During one of the hottest days of the month of July, I 881, I was called to see Mrs. W., near London Mills, in consultation with Dr. Wilson, of that place. I found her in a small bed-room, only large enough for the bed upon which she was lying, with but one window in the room. She had been confined a week previously. Her pulse was $\mathrm{I} 60$, her temperature $106^{\circ}$, the tongue dry, abdomen distended to the size of a woman at full term, the secretion of milk suppressed. She was delirious, and an odor filled the room like that of a body far gone in putrefaction.

The husband was informed that there was no probability whatever for the recovery of his wife, every symptom indicating a speedy dissolution. However, I suggested a thorough washing out of the uterus with antiseptics. A half gallon of warm water with two drachms permanganate of potash was prepared. After being placed in a proper position across the bed, a gum catheter of the largest size, with several fenestræ in the lower end, was passed up into the uterus as far as it would go. A common Davidson syringe was attached to it by means of a piece of rubber tubing. The uterus was thoroughly washed out, the injection being continued until the water returning was perfectly free from all odor and clear in color. The discharge from the uterus was of such a pecu- liarly strong odor, that it was impossible to free the hands from it. It was like that one acquires in the dissection of bodies.

Such a change as took place in this woman's case within an hour, I never before saw in any case. Immediately after this cleansing process the delirium passed off, the temperature fell, the pulse came down -in short, the effect was simply magical. She was given twenty grain doses of the sulphite of soda. The husband was taught how to administer the injections, which were to be given every three hours-one of a solution of permanganate of potash, the other of carbolized water, alternately. The patient made a speedy recovery from the severest attack of puerperal septicæmia I have ever seen.

Twelve years before this case,. in September, I 869, I was called to see a woman who had had an abortion produced by mechanical means. She had a rapid pulse, high fever, hot skin, unquenchable thirst, irregular chills, an icteric countenance, and a sanious discharge from the uterus that smelled horribly.

A half dozen intra-uterine injections of carbolized water thoroughly administered, rescued her from impending death.

Ever since that time I have used this method in all such cases with similar results with a few exceptions. They are to be regarded as similar to surgical fever, the result of decomposing débris in the uterus, which is absorbed from the uterine surface, and which Sir James Simpson has aptly compared to the flap of an amputated thigh, with its raw and bleeding surfaces, and patulous vessels ready to absorb decomposing fluids with which they may lie in contact.

The following long-continued case illustrates, in another way, the value of these intra-uterine injections. The last day of January, saw Mrs. M. A., aged 24 , living near Hermon, a strong and vigorous woman. She had been confined 24 hours previously. The child was dead when delivered, and was so much decomposed that portions of the skin slipped from the body when handled. She had had a chill and profuse flooding a few hours before I saw her. The pulse was I 40, the temperature $105^{\circ}$, skin hot, tongue dry, and abdomen tympanitic and distended to the size it was before her confinement.

The uterus was immediately washed out with carbolized water, giving great relief to the patient. The injections were kept up every three hours for a week, with the effect of reducing the temperature after every injection. Quinine was also freely administered. Thinking, perhaps, that the septic matter was sufficiently removed from the uterus, the injections were discontinued. Within 24 hours a severe chill, followed by a high temperature, announced the absorption of more septic material. The intra-uterine injections were again resorted to, with the same results, diminishing of temperature, and lowering of the pulse. At the end of a week, as there seemed to be so much improvement, they were once more discontinued. Again, in a short time, there was a chill and return of former symptoms. Again we returned to the use of the intra-uterine injections, and kept them up until every vestige of fever had disappeared from the case for several days. 
In this case there was no bad odor from the discharges, as in the former cases, and the effect of the injections in arresting the poison of the septic matter was prompt and striking.

Should antiseptics be used in every puerperal case? I believe so, especially as antisepsis has come to be considered as the use of every means of cleanliness, and the prevention of infectious or contagious matters. And just here let me record it as my fixed belief, that thousands of women have come to their deaths, from the dirt and other matters under the finger nails of their attendants. That dirt, the nest of infectious particles scraped from the body of himself, or possibly bathed in the infectious fluids of another person, is conveyed by means of the touch to the abraded surfaces in the puerperal patient, and she becomes inoculated with the virus that causes her death. I believe that any one suffering from a chronic skin disease, or who is afflicted with a chronic ulcer of any kind, should not attend upon puerperal patients, no more than he who is attending patients with scarlatina or erysipelas. In fact it is my deliberate judgment, that puerperal women should not be attended by the general practitioner at all, and for one I will gladly welcome the day, when I can turn over all such patients to well educated and thoroughly trained lady physicians who will make that their special calling.

Begging pardon for this digression I would say in conclusion that within twenty-four hours after confinement, the use of vaginal injections of carbolized water, affords the greatest comfort to the patient, bathing and soothing the neck of the uterus where there may be abrasions or lacerations, and cleansing and purifying the parts. If the discharges become foul in odor, and there is a marked rise in the temperature, then the injections should be intra-uterine. One precaution should be borne in mind, in prescribing vaginal injections, and that is to have the hole in the end of the pipe or syringe closed. I have in several cases seen a severe and dangerous uterine colic, caused by the injection passing into the uterus through the patulous and open os. In the rubber syringes that are sold for female use, I often prepare the pipe for such purposes by closing the central hole in the end, and enlarging the others in such a way that a backward current is given to the injection.

\section{IMPORTANCE OF DIAGNOSIS IN OPHTHAL- MOLOGY.}

BY E. WILliaMS, M. D., CINCINNATI, O.

Read in Section on Ophthalmology, Otology, etc., American Medical Association, May, 1884 .

If a man were seen firing in the air expecting ducks to fall without looking if any are flying over, he would be brought to court on a quest of lunacy. Why should shotgun practice in the medical profession, similarly aimless, be less criminal evidence of a fool ? Rifle practice in the heaing art requires intelligence and skill. No surer proof of a quack can be asked than a prescription containing a great variety of romantic remedies. Quacks and nostrum venders can kill just as well at long range, and with a shotgun, as with an honest, scientific weapon. The honest, educated physician insists on a personal examination before risking an opinion or prescription. Diagnosis demands knowledge, to which there is no royal road.

The prime importance of intelligent diagnosis need not be argued. Narrow notions of specialties in medicine are a great professional misfortune. How often do expectant students propose to study the eye exclusively, without the labor of a regular course of study and a degree? The only antidote to this nonsense is to insist on a good preliminary education, and a thorough study of medicine, through three to five years. If then a specialty is chosen, the choice will be discriminating. In that way only can a foundation be laid in solid rock for a special structure.

The eye is the most exquisite part of the body, and more closely linked with the healthy flow of function in the organism than any other organ. No violinist, however gifted, can become preëminent except he begin the manual training in early years. Shall less be demanded of one who is to play upon the living harp of a thousand strings, with its mixed melody of earthly and heavenly vibrations? Light, nimble and delicate manipulations are eminently required in an oculist. These can only be achieved by handy hands, early, long, and faithfully trained.

But without intelligent diagnosis, even these accomplishments go for nothing. Thorough familiarity with optics, including mathematics, is demanded if anything but mediocrity is expected. No department of ophthalmology is of more practical importance than the anomalies of refraction and accommodation. To a thorough, clear diagnosis in these, the physician must be familiar with physiological optics. The flippant way that practitioners talk about " taking up a specialty," were it not for the tragical consequences, would be simply ludicrous.

The mercenary estimate placed by the public on the faithful army of Esculapius, is due largely to failures of diagnosis and their consequences. With few exceptions, the world don't know the difference between a decent physician and professional robbers. They call them all doctors together. The only way out of this "Slough of Despond " is for all educated, decent medical gentlemen to pool their issues against the bloody and thievish-fingered quacks of all colors and persuasions.

All honor to the American Medical Association for the lofty and only safe ground of its Code of Ethics! No one who lives up to its letter and spirit can ever fall into the dirty ways of irregulars and tradesmen in our noble profession. It is the mean, mercenary spirit that eats out all bowels of mercy and scruples of conscience in a medical man. Broad intelligence and thorough honesty are the qualifications of a true physician. I state a fact. that there are too many medical colleges in this country. It is too easy for the ignorant and unscrupulous to get into the motley ranks of our profession. According to 